ఠ

\title{
Fear of falling: efficacy of virtual reality associated with serious games in elderly people
}

\author{
This article was published in the following Dove Press journal: \\ Neuropsychiatric Disease and Treatment \\ 15 April 2016 \\ Number of times this article has been viewed
}

\author{
Fanny Levy' \\ Pierre Leboucher ${ }^{2}$ \\ Gilles Rautureau ${ }^{2}$ \\ Odile Komano ${ }^{2}$ \\ Bruno Millet' \\ Roland Jouvent' \\ 'Department of Adults Psychiatry, \\ 2PRISME-Virtual Reality, ICM-A- \\ IHU, UPMC UMR_S 975, Inserm U \\ I I27, CNRS UMR 7225, GH Pitié- \\ Salpêtrière, Paris, France
}

Correspondence: Fanny Levy Pitié Salpétrière Hospital, 47-83 Boulevard de l'Hôpital, 75013 Paris, France

Tel +33 | 42 I6 2894

$\mathrm{Fax}+33$ I $42 \quad 16$ I8 26

Email fanny.levy@orange.fr
Objective: Fear of falling is defined as an ongoing concern about falling that is not explained by physical examination. Focusing on the psychological dimension of this pathology (phobic reaction to walking), we looked at how virtual reality associated with serious games can be used to treat this pathology.

Methods: Participants with fear of falling were randomly assigned to either a treatment group or a waiting list. The therapy consisted of 12 weekly sessions of virtual reality exposure therapy associated with serious games.

Results: Sixteen participants were included. The mean age of the treatment group was 72 years and that of the control group was 69 years. Participants' scores on the fear of falling measure improved after treatment with virtual reality associated with serious games, leading to a significant difference between the two groups.

Conclusion: Virtual reality exposure therapy associated with serious games can be used in the treatment of fear of falling. The two techniques are complementary (top-down and bottom-up processes). To our knowledge, this is the first time that a combination of the two has been assessed. There was a specific effect of this therapy on the phobic reaction. Further studies are needed to confirm its efficacy and identify its underlying mechanism.

Keywords: fear of falling, virtual reality exposure therapy, serious games, phobia, anxiety disorders

\section{Introduction}

Fear of falling is an intense fear of standing or walking, ${ }^{1}$ with an ongoing "concern about falling that leads to an individual avoiding activities that he/she remains capable of performing". ${ }^{2}$ Neurological examinations reveal no abnormalities that might explain the inability to walk safely on level ground. ${ }^{1}$ Previous falls are not needed to induce fear of falling. ${ }^{3,4}$

Fear of falling is frequent, with a prevalence of $24 \%-55 \%$ in elderly people. ${ }^{4-7}$ Among individuals who have experienced a fall, the prevalence rises to between $24 \%$ and $92 \%{ }^{4,5,8,9}$

Fear of falling can have serious consequences for elderly people, including accidental death, ${ }^{10}$ physical injuries, ${ }^{10,11}$ reduced quality of life, ${ }^{12-15}$ and impaired social interaction. ${ }^{14}$ The frequency of fear of falling and its adverse consequences suggest that therapeutic strategies need to be developed and assessed. According to the meta-analysis of Jung et al, ${ }^{15}$ exercise interventions, hip protectors, and combined interventions are efficient treatments for fear of falling. However, according to the meta-analysis of Rand et al, exercise and multifactorial interventions have low significant effects and tai chi has a moderately significant effect. ${ }^{16}$ Therefore, these current interventions bring only limited benefits. Moreover, these approaches focus on the balance dimension of fear of falling, without considering the psychological dimension. We suggest that fear of 
falling should actually be regarded as an anxiety disorder (phobic reaction to walking) and thus treated with cognitive behavioral therapy, which is the gold standard psychotherapeutic treatment for this kind of pathology. ${ }^{17,18}$ One type of exposure is virtual reality exposure, which has already been successfully used in the treatment of anxiety disorders. ${ }^{19-21}$ Virtual reality involves the creation of an interactive, computer-generated, three-dimensional (3D) environment. ${ }^{22}$ Studies have already used either virtual reality ${ }^{23}$ or serious games (video games, such as the Nintendo Wii with its balance board, can be used as a substitute for traditional force platforms to improve dynamic strength and balance $)^{24-27}$ to treat fear of falling. However, the results are heterogeneous. We initially tried virtual reality exposure on its own as a treatment for fear of falling. However, as patients walked carelessly after therapy and were at risk of falling, we then administered serious games as a form of augmented reality to reinstate their bodily reality and embody the new beliefs induced by the virtual reality exposure. To our knowledge, no study had previously investigated this combination of techniques. Based on the premise that fear of falling is an anxiety disorder, we postulated that combining virtual reality (used as a type of exposure) with serious games (used as an embodiment method) can be an effective treatment. Thus, the aim of our study was to assess virtual reality therapy associated with serious games in the treatment of fear of falling.

\section{Methods}

\section{Participants}

We recruited participants who exhibited a fear of falling, defined as a phobic reaction to walking. For our inclusion criteria, we applied the criterion of specific phobia: disproportioned fear in this case provoked by walking with anxious reaction (palpitations, sweat, etc) and behavioral avoidance. In somatic diseases (eg, Parkinson's disease) with potential repercussions on walking, such a fear can seem adaptive, in which case, a specialist has to assess whether the degree of fear matches the walking difficulties brought about by the current pathology. Therefore, fear of falling was diagnosed if the fear experienced while walking was prominent and disproportionate. In this sense, comorbidities involving a physical disability were not an exclusion criterion. Exclusion criteria were a current severe depressive episode (Beck Depression Inventory [BDI] $>30$ ), an inability to understand or respond to study questionnaires, severe visual impairment (inability to see the screen when wearing the helmet), and an inability to walk at all for a physical reason (eg, paraplegia). All patients provided their written informed consent. This research has been approved by the ethics committee of the University of Paris VI.

\section{Measures}

No more than a week before and after the intervention, participants underwent a nonstructured clinical assessment featuring the BDI (21-item version), Spielberger State-Trait Anxiety Inventory (state anxiety: STAI form Y-A; trait anxiety: STAI form Y-B), Sheehan Disability Scale (SDS), ${ }^{28}$ and fear of falling measure (FFM). ${ }^{29}$ The items in the FFM refer to a continuum of activities encountered in daily life, ranging from the least likely to evoke concerns about falling (eg, getting out of bed) to the most likely to do so (eg, walking outside alone when it is icy). Nineteen activities are probed: getting out of bed, getting on/off the toilet, getting dressed, taking a walk, picking up something lightweight from the floor, carrying a full plate, getting in/out of a car, walking on a crowded sidewalk, climbing up well lit stairs, climbing up poorly lit stairs, carrying bundles up well lit stairs, carry bundles up poorly lit stairs, climbing into a bus, using a step stool to reach something in a cupboard, stepping off a curb, getting in/out of a bathtub, standing on a moving bus, using an escalator, and walking outside alone when it is icy. For each item, scored from 1 to 3, participants are asked to indicate how worried they would be about performing a particular activity (very worried, moderately worried, not at all worried). Therefore, the total score ranges from 19 to 57 . In our study, the assessor was different from the therapist.

\section{Intervention}

Participants were randomly assigned to either the treatment group or a waiting list control group (by drawing lots).

The therapy consisted of 12 sessions, each lasting $\sim 40$ minutes, composed of a brief clinical interview and virtual reality exposure followed by a serious game. Sessions were scheduled once a week. For the virtual reality component, participants were asked to walk in $3 \mathrm{D}$ virtual worlds. They sat on a swivel chair. Participants wore a V8 head-mounted display (Virtual Research Systems Inc., Aptos, CA, USA) that allowed them to be immersed in the virtual world, where they were able to walk using a wireless mouse. The actual exposure to virtual reality lasted $\sim 15$ minutes. There were four different virtual worlds of increasing difficulty (city, castle, island, underground). In the first world (city), the ground was level, with little variation (sidewalk). In the castle, there was less space to walk (corridor) and a high step. In the island world, the ground was uneven, with rocks and differences in height, while in the final, underground world, participants had to go up and 
down steep flights of steps. A desktop personal computer (PC) (Transtec - Intel ${ }^{\circledR}$ Core $^{\mathrm{TM}}$ i7-960 Processor 3.20 GHz) and a Nvidia GeForce GTX 470 graphics card with 1 GB of memory were used to display the virtual worlds in a $640 \times 480$ resolution stereoscopic (3D) format. A 3D electromagnetic sensor (Nest of Birds; Ascension Technology Corporation, Milton, VT, USA) was fixed to the helmet to measure head and body motion during navigation. The serious game component lasted $\sim 15$ minutes. We used the EyeToy interface for PlayStation 2 (Sony, Tokyo, Japan) and a video projector to display the image on a large $2 \mathrm{~m} \times 1.5 \mathrm{~m}$ screen. Motion was detected by a USB camera. Participants played the video games by moving their bodies. The goal was to make the participants move and give them visual feedback on their movements. We administered two different games. In the first one, participants were asked to wash a window covered in foam. They had to raise their hands above their head and move them from side to side to remove all the foam. In the second game, participants had to use their hands to get rid of enemies coming from the left and right (kung fu). The level of difficulty was adjusted to match each participant's progress (parameter: time taken to wash the window for the first game and rapidity and number of enemies neutralized for the second one).

The control participants remained on the waiting list for 12 weeks, during which time there was no change in their treatment.

\section{Statistical analysis}

We calculated the differences in the scores on the various scales (FFM, BDI, STAI-Y-A, STAI-Y-B, and SDS) before and after the intervention for the virtual reality therapy and control (waiting list) groups. Nonparametric tests (Mann-Whitney and Fisher) were used to compare these differences between these two groups. The primary outcome was the difference in FFM scores.

\section{Results}

We recruited 16 participants with fear of falling: nine in the treatment group and seven in the control group. Six women and three men were included in the treatment group, and four women and three men in the control group (no significant difference, $P=1.00$ ). Mean ages (standard deviation [SD]) were 72.4 years $(12.25)$ for the treatment group and 68.65 years (19.05) for the control group. There was no significant difference between the two groups for age $(P=0.634)$. All the participants exhibited fear of falling and had walking difficulties that could not be explained by their physical condition. Their fear of falling had emerged following a fall (traumatic psychological origin) for all participants. They were all able to walk with or without mechanical help. One participant in each group had sustained a fracture owing to a fall. No significant difference was found at baseline on the mean FFM scores: 39.0 (7.71) for the treatment group and 34.43 (9.32) for the control group ( $P=0.351)$. Participants were moderately to severely impaired in their social and family lives because of their fear of falling. Mean SDS scores for social life were 6.44 (3.17) for the treatment group and 6.00 (1.15) for the control group. Mean SDS scores for family life were 5.44 (4.07) and 4.71 (3.40), respectively. No significant difference was found between the two groups $(P=0.485$ and $P=0.709)$. Participants presented several comorbidities, but these could not explain their walking difficulties. They included systemic sclerosis (one in each group), Parkinson's disease (two in the treatment group), knee prosthesis for arthrosis (one in each group), and cerebral stroke (two in the treatment group: one with vertebrobasilar stroke and one with Sylvian fissure stroke). None of the participants had severe depression: mean BDI scores were 12.88 ( $\mathrm{SD}=7.00)$ for the treatment group and 8.29 (5.82) for the control group. Mean STAI-Y-A scores were 39.44 (14.64) for the treatment group and 35.29 (8.18) for the control group. Mean STAI-Y-B scores were 47.5 (9.29) for the treatment group and 42.57 (7.04) for the control group. No significant difference was found between the two groups at baseline for these measures: BDI $(P=0.266)$, STAI-Y-A $(P=0.873)$, and STAI-Y-B $(P=0.164)$. Baseline results are set out in Table 1.

The difference between mean FFM scores before and after the intervention was -2.78 (4.82) for the therapy group and 4.14 (4.3) for the control group, with a significantly

Table I Participants' mean (SD) baseline characteristics

\begin{tabular}{llll}
\hline Characteristics & VRET $(\mathbf{n}=\mathbf{9})$ & WL $(\mathbf{n}=\mathbf{7})$ & $P$-value \\
\hline $\begin{array}{l}\text { Age, years } \\
\text { Sex }\end{array}$ & $72.4(I 2.25)$ & $68.65(19.05)$ & 0.634 \\
$\quad$ Female & 6 & 4 & \\
$\quad$ Male & 3 & 3 & 1.000 \\
FFM & $39(7.7 I)$ & $34.43(9.32)$ & $0.35 I$ \\
BDI & I2.88(7.00) & $8.29(5.82)$ & 0.266 \\
STAI & & & \\
$\quad$ Y-A & $39.44(14.64)$ & $35.29(8.18)$ & 0.873 \\
$\quad$ Y-B & $47.5(9.29)$ & $42.57(7.04)$ & 0.164 \\
SDS & & & \\
$\quad$ Social life & $6.44(3.17)$ & $5.44(4.07)$ & 0.485 \\
$\quad$ Family life & $6.00(I .15)$ & $4.7 I(3.40)$ & 0.709 \\
\hline
\end{tabular}

Abbreviations: VRET, virtual reality exposure therapy; WL, waiting list; FFM, fear of falling measure; BDI, Beck Depression Inventory; STAI, Spielberger State-Trait Anxiety Inventory (state anxiety: STAI form Y-A; trait anxiety: STAI form Y-B); SDS, Sheehan Disability Scale; SD, standard deviation. 
Table 2 Comparison of mean (SD) results for VRET and WL groups

\begin{tabular}{llll}
\hline Scale & VRET & WL & P-value \\
\hline FFM & $-2.78(4.82)$ & $4.14(4.3)$ & $0.007^{*}$ \\
BDI & $-3.00(10.79)$ & $3.25(2.75)$ & 0.476 \\
STAI & & & \\
$\quad$ Y-A & $-8.86(14.46)$ & $9.80(4.66)$ & $0.005^{*}$ \\
Y-B & $-5.50(9.18)$ & $1.60(9.76)$ & 0.247 \\
SDS & & & \\
$\quad$ Social life & $-1.22(2.16)$ & $0.57(2.30)$ & 0.180 \\
Family life & $1.56(3.78)$ & $1.29(3.77)$ & 0.120 \\
\hline
\end{tabular}

Note: $* P<0.05$.

Abbreviations: VRET, virtual reality exposure therapy; WL, waiting list; FFM, fear of falling measure; BDI, Beck Depression Inventory; STAI, Spielberger State-Trait Anxiety Inventory (state anxiety: STAI form Y-A; trait anxiety: STAI form Y-B); SDS, Sheehan Disability Scale; SD, standard deviation.

greater decrease in the therapy group $(P=0.007)$. The difference in mean BDI scores between the therapy and control groups was not significant: $-3.00(10.79)$ versus 3.25 (2.75) $(P=0.476)$. The difference between the two groups on mean state anxiety scores (STAI-Y-A) was significant: -8.86 (14.46) versus 9.8 (4.66) $(P=0.005)$. The difference between the two groups on mean trait anxiety scores (STAI-Y-B) was not significant: -5.50 (9.18) versus 1.60 (9.76), $P=0.247$. The difference between the two groups on mean SDS scores for social life was not significant: -1.22 (2.16) versus 0.57 (2.30), $P=0.180$. Nor was the difference in mean SDS scores for family life: $-1.56(-3.78)$ versus 1.29 (3.77), respectively; $P=0.120$. The results are shown in Table 2 .

\section{Discussion}

In this study, we assessed the efficacy of associating virtual reality therapy with serious games to treat fear of falling. The FFM scores of participants who completed the 12 sessions were significantly lower than those of participants who were treated as usual. Therefore, virtual reality therapy associated with serious games can be used in the treatment of fear of falling.

This study was innovative for two reasons. First, we combined two complementary techniques: virtual reality therapy and serious games. To our knowledge, this is the first time that this combination has been assessed. Virtual reality provides real-time feedback ${ }^{30}$ and requires the cognitive organization and execution of synchronized motion (topdown process), whereas serious games allow for embodiment (bottom-up process) and thus complement virtual reality. Second, in contrast to previous trials that highlighted the balance dimension, we focused on the psychological dimension (phobic reaction) of this pathology, with an intervention involving virtual exposure to walking with no vestibular rehabilitation. Thus, the fear of falling was regarded as an anxiety disorder that was treated with exposure therapy in the form of virtual reality, whose validity has already been proved in this kind of pathology.

We did not find any significant improvement in BDI, STAI-Y-B, or SDS scores in the wake of the virtual reality therapy. Therefore, the improvement in fear of falling was not mediated by an effect on patients' depressive mood (BDI), general functioning (STAI-Y-B), or motor aspects (SDS). Thus, the effect of virtual reality therapy associated with serious games on the fear of falling is specific and concerns the phobic reaction itself.

Participants' mean ages were 69 years (virtual reality) and 72 years (control), indicating that new technologies can be used efficiently with elderly people.

\section{Limitations}

This study had several limitations. The number of participants was, of course, small. Furthermore, because the randomization was not done with the appropriate software, but by drawing lots, it cannot be regarded as a true randomized clinical trial.

\section{Conclusion}

We can draw several conclusions from this preliminary analysis. First, results suggest that virtual reality therapy is an effective treatment for fear of falling syndrome. However, because we did not use a proper randomization procedure, we are planning to undertake a true randomized control trial featuring the same treatment protocol (virtual reality combined with serious games). Second, our preliminary results indicate that 221 patients would have to be included in such a trial (alpha risk: 0.05 ; beta risk: 0.2 ) ${ }^{31}$ This number of patients could be reduced by applying an analysis of variance to the baseline scores. ${ }^{32}$ Increasing the number of patients would probably lead to a more clearly significant difference at the end point. By including sufficient patients to achieve statistical power, we would be able to reach a definitive conclusion as to the usefulness of virtual reality therapy associated with serious games for treating fear of falling.

\section{Disclosure}

The authors report no conflicts of interest in this work.

\section{References}

1. Bhala RP, O'Donnell J, Thoppil E. Ptophobia. Phobic fear of falling and its clinical management. Phys Ther. 1982;62:187-190. 
2. Tinetti ME, Powell L. Fear of falling and low self-efficacy: a case of dependence in elderly persons. J Gerontol. 1993;48 Spec No:35-38.

3. Maki BE, Holliday PJ, Topper AK. Fear of falling and postural performance in the elderly. J Gerontol. 1991;46:M123-M131.

4. Howland J, Peterson EW, Levin WC, Fried L, Pordon D, Bak S. Fear of falling among the community-dwelling elderly. J Aging Health. 1993;5: 229-243.

5. Howland J, Lachman ME, Peterson EW, Cote J, Kasten L, Jette A. Covariates of fear of falling and associated activity curtailment. Gerontologist. 1998;38:549-555.

6. Tinetti ME, Mendes de Leon CF, Doucette JT, Baker DI. Fear of falling and fall-related efficacy in relationship to functioning among community-living elders. J Gerontol. 1994;49:M140-M147.

7. Zijlstra G, van Haastregt JC, van Eijk JT, van Rossum E, Stalenhoef PA, Kempen GI. Prevalence and correlates of fear of falling, and associated avoidance of activity in the general population of community-living older people. Age Ageing. 2007;36:304-309.

8. Vellas BJ, Wayne SJ, Romero LJ, Baumgartner RN, Garry PJ. Fear of falling and restriction of mobility in elderly fallers. Age Ageing. 1997;26: 189-193.

9. Aoyagi K, Ross PD, Davis JW, Wasnich RD, Hayashi T, Takemoto T. Falls among community-dwelling elderly in Japan. J Bone Miner Res. 1998; 13:1468-1474.

10. Fuller GF. Falls in the elderly. Am Fam Physician. 2000;61(2159-2168): 2173-2174.

11. Stel VS, Smit JH, Pluijm SMF, Lips P. Consequences of falling in older men and women and risk factors for health service use and functional decline. Age Ageing. 2004;33:58-65.

12. Lachman ME, Howland J, Tennstedt S, Jette A, Assmann S, Peterson EW. Fear of falling and activity restriction: the survey of activities and fear of falling in the elderly (SAFE). J Gerontol B Psychol Sci Soc Sci. 1998;53:43-50.

13. Lawrence RH, Tennstedt SL, Kasten LE, Shih J, Howland J, Jette AM. Intensity and correlates of fear of falling and hurting oneself in the next year: baseline findings from a Roybal Center fear of falling intervention. J Aging Health. 1998;10:267-286.

14. Arfken CL, Lach HW, Birge SJ, Miller JP. The prevalence and correlates of fear of falling in elderly persons living in the community. $\mathrm{Am}$ J Public Health. 1994;84:565-570.

15. Jung D, Lee J, Lee S-M. A meta-analysis of fear of falling treatment programs for the elderly. West J Nurs Res. 2009;31:6-16.

16. Rand D, Miller WC, Yiu J, Eng JJ. Interventions for addressing low balance confidence in older adults: a systematic review and meta-analysis. Age Ageing. 2011;40:297-306.

17. Olatunji BO, Cisler JM, Deacon BJ. Efficacy of cognitive behavioral therapy for anxiety disorders: a review of meta-analytic findings. Psychiatr Clin North Am. 2010;33:557-577.
18. Watts SE, Turnell A, Kladnitski N, Newby JM, Andrews G. Treatmentas-usual (TAU) is anything but usual: a meta-analysis of CBT versus TAU for anxiety and depression. J Affect Disord. 2015;175:152-167.

19. Opriş D, Pintea S, García-Palacios A, Botella C, Szamosközi Ş, David D. Virtual reality exposure therapy in anxiety disorders: a quantitative meta-analysis. Depress Anxiety. 2012;29:85-93.

20. Parsons TD, Rizzo AA. Affective outcomes of virtual reality exposure therapy for anxiety and specific phobias: a meta-analysis. J Behav Ther Exp Psychiatry. 2008;39:250-261.

21. Powers MB, Emmelkamp PMG. Virtual reality exposure therapy for anxiety disorders: a meta-analysis. J Anxiety Disord. 2008;22:561-569.

22. Riva G, Mantovani F, Capideville CS, et al. Affective interactions using virtual reality: the link between presence and emotions. Cyberpsychol Behav. 2007;10:45-56.

23. Duque G, Boersma D, Loza-Diaz G, et al. Effects of balance training using a virtual-reality system in older fallers. Clin Interv Aging. 2013;8: 257-263.

24. Pietrzak E, Cotea C, Pullman S. Using commercial video games for falls prevention in older adults: the way for the future? J Geriatr Phys Ther. 2014;37:166-177.

25. Pluchino A, Lee SY, Asfour S, Roos BA, Signorile JF. Pilot study comparing changes in postural control after training using a video game balance board program and 2 standard activity-based balance intervention programs. Arch Phys Med Rehabil. 2012;93:1138-1146.

26. Williams MA, Soiza RL, Jenkinson AM, Stewart A. EXercising with Computers in Later Life (EXCELL) - pilot and feasibility study of the acceptability of the Nintendo ${ }^{\circledR}$ WiiFit in community-dwelling fallers. BMC Res Notes. 2010;3:238.

27. Jorgensen MG, Laessoe U, Hendriksen C, Nielsen OBF, Aagaard P Efficacy of Nintendo Wii training on mechanical leg muscle function and postural balance in community-dwelling older adults: a randomized controlled trial. J Gerontol A Biol Sci Med Sci. 2013;68:845-852.

28. Sheehan DV, Harnett-Sheehan K, Raj BA. The measurement of disability. Int Clin Psychopharmacol. 1996;11(suppl 3):89-95.

29. Velozo CA, Peterson EW. Developing meaningful Fear of Falling Measures for community dwelling elderly. Am J Phys Med Rehabil. 2001; 80:662-673

30. Cikaljo I, Matjacic Z. Advantages of virtual reality technology in rehabilitation of people with neuromuscular disorders. Recent Adv Biomed Eng. 2009;16:301-320.

31. Schwartz D, Flamant R, Lellouch J. L'essai therapeutique chez l'Homme. Paris: Flammarion; 1993.

32. Borm GF, Fransen J, Lemmens WAJG. A simple sample size formula for analysis of covariance in randomized clinical trials. JClin Epidemiol. 2007;60:1234-1238.
Neuropsychiatric Disease and Treatment

\section{Publish your work in this journal}

Neuropsychiatric Disease and Treatment is an international, peerreviewed journal of clinical therapeutics and pharmacology focusing on concise rapid reporting of clinical or pre-clinical studies on a range of neuropsychiatric and neurological disorders. This journa is indexed on PubMed Central, the 'PsycINFO' database and CAS,

\section{Dovepress}

and is the official journal of The International Neuropsychiatric Association (INA). The manuscript management system is completely online and includes a very quick and fair peer-review system, which is all easy to use. Visit http://www.dovepress.com/testimonials.php to read real quotes from published authors. 